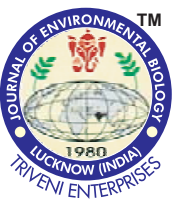

\title{
Optimization of ethanol production using pretreated corn cob and sugarcane bagasse hydrolysate by Candida parapsilosis strain BKR1
}

Authors Info

Balakrishnaraja R. ${ }^{\text {** }}$,

Balasubramanian S. ${ }^{2}$, Aravindan $P^{2}$, Arulraj A. ${ }^{2}$, Selvapriya K. ${ }^{2}$, Geethadevi S. and Nisha Rao

${ }^{1}$ Bannari Amman Institute of Technology,

Sathyamangalam-638 401, India

${ }^{2}$ Government College of Technology, Coimbatore-641 013, India

Department of Biotechnology,

Bannari Amman Institute of

Technology,

Sathyamangalam-638 401, India

*Corresponding Author Email : balakrishanrajar@bitsathy.ac.in

Key words

Candida sp.

Corn cob hydrolysate

Ethanol

Lignocelluloses

Sugarcane hydrolysate

Publication Info

Paper received : 23.05.2016

Revised received : 29.11.2016

Re-revised received : 16.03 .2017

Accepted : 23.03.2017

\section{Abstract}

Aim : Bioethanol is a suitable and best alternative to the replenishing fossil fuel in the fast growing world. To meet the rising demand of ethanol in the global market, there needs to be lot of improvisation in the existing process. One such method is to utilize more pentose sugar along with hexose in the substrate for better yield. In the present study, the efficiency of Candida parapsilosis BKR1 species for the production of ethanol was studied in corncobs and sugarcane bagasse.

Methodology : The sugarcane bagasse and corn cobs were collected from local market, Coimbatore and Candida parapsilosis BKR1 was isolated from sugar cane extract. The lignocellulosic materials were treated by various combination of pretreatment (acid and alkali pretreatment) and hydrolysis (acid, alkaline and sodium carbonate hydrolysis) methods to maximize the fermentable sugars in the substrate. The fermentation conditions such as $\mathrm{pH}$, inoculum age, nitrogen source and fermentation time for ethanol production were optimized and the fermented media of both substrates were subjected to distillation to find ethanol percentage by gas chromatography.

Results : Acid pretreatment with sodium carbonate hydrolysis produced higher sugar yield for both the lignocellulosic materials. The studies on optimization showed maximum production using $24^{\text {th }} \mathrm{hr}$ inoculum, with $1 \%$ nitrogen source at $\mathrm{pH} 6$, during $6^{\text {th }}$ day of fermentation @ $120 \mathrm{rpm}$ for corn cobs and sugarcane bagasse. The results revealed that ethanol concentration in sugarcane bagasse and corn cobs were 27.93 $\mathrm{gl}^{-1}$ and $20.78 \mathrm{gl}^{-1}$, respectively.

Interpretation : The Candida parapsilosis BKR1 strain efficiently utilized sugarcane bagasse than corn cobs and produced high concentration of ethanol.

1. Milled agro waste residues
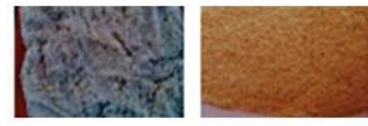

4. Ethanol Quantification : GC chromatogram

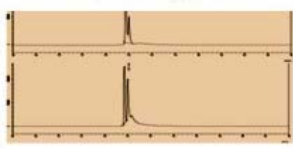

2. Pretreatment strategies

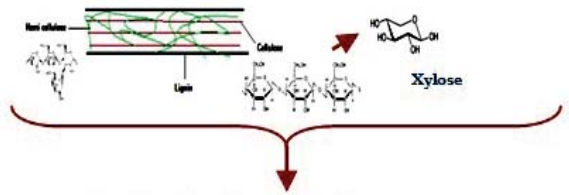

3. Optimization studies for ethanol production

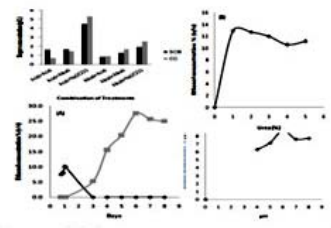

Production \& Optimization of Ethanol from agro waste residues by Candida parapsilosis BKR1 


\section{Introduction}

The demand for alternative fuel is increasing day by day due to the depletion of fossil fuels and high $\mathrm{CO}_{2}$ emission. Ethanol, a major component of worldwide gasoline market is a key for the above considered problems. Although energy equivalent of ethanol is lesser than petroleum fuel, recent trends in the technologies raises the demand for ethanol production (Mussatto etal., 2010).

Lignocellulosic biomass, such as sugarcane bagasse/ corn cob, can be economical and easily available source used for the biofuel production. Bioethanol production from lignocellulosic biomass rich in hemicellulose comprises the hydrolysis of complex sugar followed by reduced sugar fermentation. The production of ethanol from renewable biomass such as sugarcane beets, corn, cassava, potatoes, paper and agricultural waste, converting the carbohydrate enriched lignocellulosic materials into ethanol by various micro organisms (Lin et al., 2006; Ray et al., 2008).

Most typical microorganisms used for the production of bioethanol are Saccharomyces cerevisiae, Zymomonas mobilis and Escherichia coli (Lin et al., 2006) which are capable of fermenting hexose sugars/ sucrose. Most of the ethanol fermenting organism source is limited to hexose sugars. Pentose fermenting organisms are restricted to Pichiastipitis, Candida shehatae, Pachysolen tannophilus (Martiniano et al., 2013). Finding new microbial isolates from the natural sources can enable effective fermentation of pentose sugars to yield ethanol (Mussatto et al., 2010). The consistent results in optimization studies are essential to develop commercially significant process with sustainable solution. The different types of ethanol producing strains such as Saccharomyces cerevisiae, Zygosaccharomyces sp., Saccharomyces ellipsoids, Schizosaccharomyces pombe and Schizosaccharomyces mallaeri were optimized for maximum ethanol production (Osman et al., 2011).

The strain Candida parapsilosis BKR1 used in the present study was isolated from sugar cane extract and studied for the ability of utilizing sugarcane bagasse and corn cobs for the production of ethanol. The substrates used in the present investigation are agricultural waste residues obtained from sugarcane processing plant and corn fields.

\section{Materials and Methods}

Sample collection :Candida parapsilosis strain BKR1, isolated from sugar cane extracts at Sathyamangalam, Tamilnadu, was used for ethanol production. Biomass such as sugarcane bagasse (SCB) and corn cobs (CC) required for fermentation were collected from the local market, Coimbatore, Tamil Nadu and all other chemicals were of analytical grade.

Microbial inoculum preparation : Isolated Candida parapsilosis strain BKR1 was sub cultured in xylose rich medium containing $20 \mathrm{~g}$ of xylose supplemented with Yeast extract $(5 \mathrm{~g})$ and Peptone $(10 \mathrm{~g})$. The culture was inoculated into $150 \mathrm{ml}$ conical flask containing $10 \mathrm{ml}$ of medium and agitated at $200 \mathrm{rpm}$ at $30^{\circ} \mathrm{C}$ for $24 \mathrm{hr}$. After $24 \mathrm{hrs}$ of incubation, culture was centrifuged at $2200 \mathrm{~g}$ for $15 \mathrm{~min}$. The collected cells were washed with sterile distilled water.A suspension was prepared with the cell mass in sterile distilled water and utilized as inoculum.

Pretreatment of lignocellulosic materials : Sugarcane bagasse (SCB) and corn cobs (CC) were washed, shadow dried and their size were reduced. Further treatment involved delignification and hydrolysis of SCB and CC which were done separately by acid and alkali.

Dried and chopped sugarcane bagasse and corn cobs were immersed in sulfuric acid in 1:10 (w/v) ratio and next to this process, biomass were similarly subjected to alkaline treatment with $1 \mathrm{~N} \mathrm{NaOH}$ solution in 1:10 (w/v) ratio. These mixtures were hydrolysed at $121^{\circ} \mathrm{C}$ for $15 \mathrm{~min}$. The obtained hydrolysates were pressed through cheese cloth and solid biomass was taken for further hydrolysis.

These pretreated samples were further hydrolysed by $0.5 \% \mathrm{H}_{2} \mathrm{SO}_{4}$ solution, $0.5 \mathrm{~N} \mathrm{NaOH}$ and $1 \mathrm{~N} \mathrm{Na}_{2} \mathrm{CO}_{3}$ separately at $121^{\circ} \mathrm{C}$ for $20 \mathrm{~min}$. The hydrolysates were analyzed for reducing sugar concentration. Among these samples, hydrolysates with highest sugar concentration were selected for fermentation process.

Analysis of sugars and ethanol: The concentration of reducing sugar was determined by 3,5-dinitrosalicylic method (Miller, 1959). The ethanol concentration was determined by potassium dichromate method and gas chromatography method using Rtx-5 column (30 m, $0.32 \mathrm{ID}$ ) and nitrogen carrier gas $\left(53.9 \mathrm{ml} \mathrm{min}^{-1}\right)$. Injection and FID temperatures were $150^{\circ} \mathrm{C}$ and $200^{\circ} \mathrm{C}$, respectively. The temperature of oven was maintained at $325^{\circ} \mathrm{C}$.

The concentration of ethanol was calculated by the following formula (Cachet, 2011):

$$
\mathrm{Ca}=[\text { Cstd *As] / Astd }
$$

Where, $\mathrm{C}_{\mathrm{a}}$ is the concentration of analyte in the sample; $\mathrm{C}_{\mathrm{st}}$ is the concentration of analyte in standard; $A_{a}$ is the area of analyte from the sample chromatogram and $A_{s t}$ is the area of analyte from the standard chromatogram.

Optimization of fermentation conditions : Many strategies are involved in order to maximize ethanol production. These strategies include both pretreatment methods and the fermentation conditions. The present study focused on the fermentation to obtain maximum concentration of ethanol - some of these conditions include age of inoculum, fermentation duration, nitrogen source and $\mathrm{pH}$ of fermentation medium. The 
Table 1: Various combinations of pretreatments processed on the raw materials-Sugarcane Bagasse (SCB) and Corn Cops (CC)

\begin{tabular}{llll}
\hline Process & Raw Material & Delignification & Hydrolysis \\
\hline Run 1 & SCB & Acid & Acid \\
Run 2 & SCB & Acid & NaOH \\
Run 3 & SCB & Acid & Sodium Carbonate \\
Run 4 & SCB & Alkali & Acid \\
Run 5 & SCB & Alkali & NaOH \\
Run 6 & SCB & Alkali & Sodium carbonate \\
Run 7 & CC & Acid & Acid \\
Run 8 & CC & Acid & NaOH \\
Run 9 & CC & Acid & Sodium Carbonate \\
Run 10 & CC & Alkali & Acid \\
Run 11 & CC & Alkali & $\mathrm{NaOH}$ \\
Run 12 & CC & Alkali & Sodium carbonate \\
\hline
\end{tabular}

optimized studies were done in pure xylose substrate and the optimized conditions were taken for raw material fermentation.

Effect of inoculum age : Candida parapsilosis BKR1 strain was taken in xylose supplemented medium and kept at $200 \mathrm{rpm}$, $30^{\circ} \mathrm{C}$. The effect of age of inoculum was studied by taking cultures at various time intervals such as 18, 20, 22, 24 and $26 \mathrm{hrs}$ and inoculated in xylose containing medium. Samples were taken at regular intervals and analyzed for ethanol concentration.

Effect of $\mathrm{pH}$ : The $\mathrm{pH}$ of xylose medium was set from 4 to 8 by adjusting with $1 \mathrm{~N} \mathrm{HCl}$ and $1 \mathrm{~N} \mathrm{NaOH}$. The optimized age of inoculum culture was taken and inoculated for all medium ranging from $\mathrm{pH} 4$ to $\mathrm{pH} 8$ and samples were analyzed for ethanol concentration at regular intervals.

Effect of fermentation time: The xylose containing medium with optimized $\mathrm{pH}$ and age of inoculum was taken for fermentation. The ethanol concentration was analyzed at regular interval from 0-6 days.

Effect of nitrogen source : The effect of urea in ethanol production was studied by varying the percentage of urea $(1 \%$, $2 \%, 3 \%, 4 \%$ and $5 \%$ nitrogen source $(10: 1 \mathrm{v} / \mathrm{v})$ in the fermentation medium.

Substrate fermentation : The supernatant from treated hydrolyzates were used as fermentation medium and optimized conditions from the optimization studies were taken for further process. $1 \%(\mathrm{v} / \mathrm{v})$ of optimized inoculum was added and the medium was kept at $37^{\circ} \mathrm{C}$ with $120 \mathrm{rpm}$ and fermented for a period of 6 days. After fermentation, the cells were separated by centrifuging and supernatant taken for distillation process. The distillates were analyzed for ethanol concentration by Gas Chromatography.

\section{Results and Discussion}

Treatment of lignocelluloses materials : The lignocellulosic

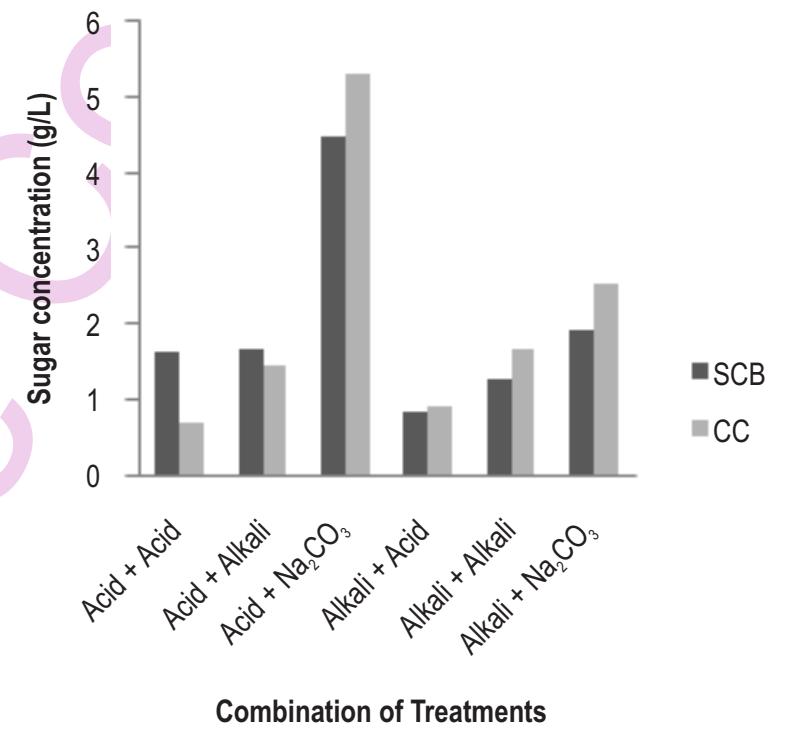

Fig. 1 : Reducing sugar concentration of pretreated and hydrolyzed samples of sugarcane bagasse (SCB) and corn cobs (CC)

materials are composed of cellulose, hemicellulose, lignin, extractives and several inorganic materials. Among these the cellulose and hemicelluloses which is made up of sugars has to be separated and hydrolysed for further fermentation process. To achieve this, the lignocellulosic materials were degraded and delignified to release cellulose and hemicelluloses components, which were further hydrolysed to release the reducing sugars. The results of pretreatments are shown in Fig. 1. Acid along with $\mathrm{Na}_{2} \mathrm{CO}_{3}$ treatment of lignocellulosic materials was found to release maximum sugar concentration as compared to other treatment procedures. The maximum sugar substrate release was found for acid with $\mathrm{Na}_{2} \mathrm{CO}_{3}$ treatment combination Size reduction of raw material decreased the polymerization of cellulosic content and also increased the accessible surface area of the material for further degradation by physiological and 
microbial reactions.

In sugarcane bagasse (SCB) residual pretreatment, run 1,2 and 3 had high sugar yield when compared with that of than 4 , 5 and 6 which indicates that acid pretreatment gave high sugar yield than alkali treatment but the results were contrast in corncob (CC) residues, as both acid and alkali treatment gave more similar amount of reducing sugars. These pretreated samples were further hydrolyzed by acid and alkali (Sodium hydroxide and
Sodium carbonate). Among the methods tried, acid pretreated with sodium carbonate hydrolysis (Run 3 \& 9) yielded high reducing sugars, followed by alkali with sodium carbonate (Run 6 \& 12) than other process. It suggests that the acid pretreatment might completely solubilize hemicelluloses and gives better hydrolysable substrates. Verardi et al. (2016) showed that the yields of monosaccharide's from a lignocellulosic biomass is complex but economical for monosaccharides such as xylose,
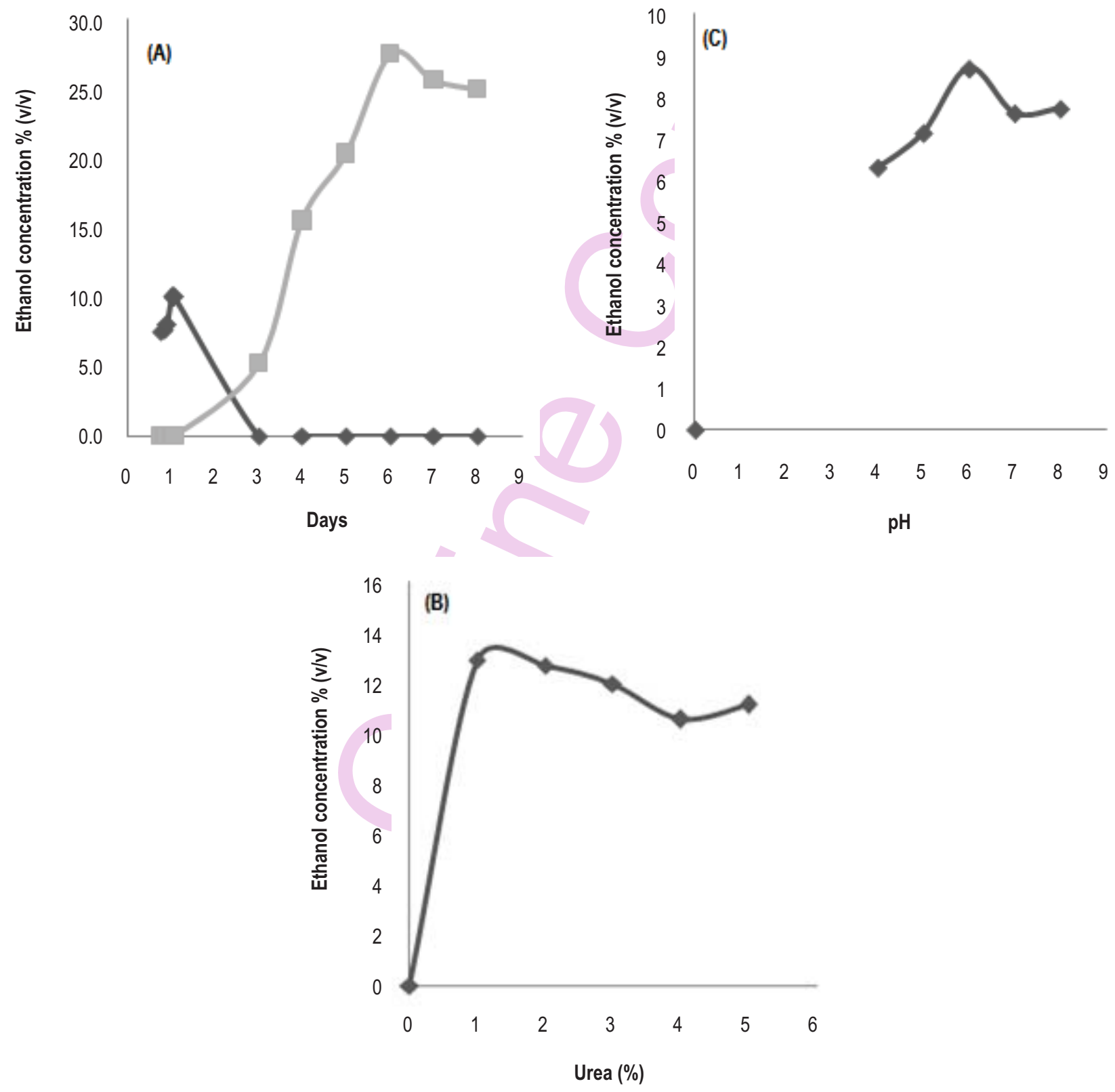

Fig. 2: Effect of parameters on ethanol production from xylose (A) fermentation time ( $\square$ ) and age of inoculums (४); (B) pH; (C) nitrogen source 
arabinose, glucose and mannose.

Sodium carbonate hydrolysis yields more sugar than other hydrolysis methods mainly due to hydrophilic acid dissolvable nature of sodium carbonate. The results are contrary to Bjerre et al., (1996) who stated that addition of sodium carbonate dint has much effect on hemicellulose conversion. These might be due to inhibition of degradation of sugars to byproducts like furfural which will decrease the sugar concentration. Due to the above mentioned reasons, the interference between undesired byproducts and enhances the hemicellulose conversion from agro waste residues.Sodium carbonate might act as detoxifier to prevent degradation of products and also hydrolyze the product. Also the sugar concentration was found to be maximum $\left(>5 \mathrm{gl}^{-1}\right)$ for sugarcane bagasse compared to corncob residue probably due to interference effect of furfural derivatives in $\mathrm{CC}$ residues.

\section{Optimization Studies}

Effect of inoculum age : The growth of Candida parapsilosis $B K R 1$ and simultaneous production of ethanol mainly depends on the production of sugars from $C C$ and $S C B$ residues as substrate. The inoculum age of Candida parapsilosis BKR1 varied from 18 to $26 \mathrm{hrs}$ ( 0.75 to 1.08 days). Fig.2A shows the effect of inoculum age on ethanol production. A decrease in the inoculum age leads to decreased production and the 24 hrs slant showed higher production of ethanol. Hence, it is recommended to use $24 \mathrm{hrs}$ slant culture of Candida parapsilosis BKR1 for economical production of ethanol. The optimized $24 \mathrm{hr}$ microbial culture was able to yield maximum ethanol production due to the availability of majority of active viable microbes in their log phase. These log phase cultures ensures rapid growth and enhance the ethanol productivity. Further rise in the inoculum age also resulted in same ethanol yield of $10 \%$.

Effect of $\mathrm{pH}$ : Among the various $\mathrm{pH}$ of fermentation medium tried, the ethanol production was observed between $\mathrm{pH}$ 4-8 (Fig.2B). The maximum ethanol was obtained at $\mathrm{pH} 6$ and no production of ethanol was observed below $\mathrm{pH} 4$ (data not shown). These results suggested that maintaining intracellular $\mathrm{pH}$ is most vital for the proper functioning of glycolytic enzymes in yeast. Variation in the mid pH level creates ionic imbalance across the microbial cell wall which builds up stress and further inhibits yeast metabolic pathway for ethanol production. High acidic condition of the solution due to organic acids tends to reduce the microbial growth. Naturally by increasing the $\mathrm{pH}$ of fermentation medium, the microbial growth is inhibited and eventually the broth is flooded with more of organic acids (Graves et al., 2006; Izmirlioglu and Demirci, 2012; Turhan et al., 2010; Osman et al., 2011). However these results were in contrast to Du preez et al., (1986) who concluded that Candida was insensitive to $\mathrm{pH}$ range of $2.5-6.5$ for the production of ethanol. The ability of the Candida species to produce ethanol was reported at $\mathrm{pH}>6$ as $8.69 \%$ (v/v).
In the neutral and alkaline condition the ethanol yield were drastically reduced might be due to formation of salt when an unstable sugar reacts with alkali content in excess (Graves et al., 2006).

Effect of fermentation time : The ethanol concentrations were analyzed from zero hour and there was no ethanol production for first 48 hrs (data not shown). This might be due to the carbon utilization for the cellular maintenance and production of xylitol based on their metabolic pathways. The production of xylitol was initiated at $48 \mathrm{hrs}$ and reached maximum at $72 \mathrm{hrs}$. After $72 \mathrm{hrs}$, the xylitol concentration decreased with initiation of ethanol production. The concentration of ethanol started increasing from $72 \mathrm{hrs}$ and gave maximum yield at $144 \mathrm{hrs}$ (Fig.2A). The optimum ethanol yield was found to be $27.5 \%(\mathrm{v} / \mathrm{v})$ on $6^{\text {th }}$ day. The probable reason for producing ethanol after 144 hrs is due to delayed metabolic pathways and accumulation of xylitol in the previous stages. The rate limiting step in the pentose phosphate pathway may be overcome by enhancing the redox stress induced during xylitol formation inside the microbial cell. Itelima et al., (2013), concludes that the accumulation of xylitol enhances the redox potential inside the microbial cell which ultimately determines the rate of ethanol production, which is in line with the present investigation results.

Effect of nitrogen source : The organic nitrogen plays a major role in the growth of yeast cells and production of ethanol (Jeffries, 1985). Among the various sources and concentrations selected, $1 \%$ of urea in fermentation medium gave high ethanol concentration of $13 \%(\mathrm{v} / \mathrm{v})$, which is depicted in Fig.2C. However, Nofemele et al., (2012) and Chan-u-tit et al., (2013) reported that the increasing percentage of urea decreased the production of ethanol. The reason for the reduction in ethanol production is probably due to the inhibition/ partial blockage of oxidoreductive enzymes involved in the pentose phosphate pathway. The ratio between NAD/ NADPH - linked xylose reductase plays a major role in determining the utilization of nitrogen source, supplied along with the microbial medium supplement. It is assumed that the optimum concentration of nitrogen source rapidly demands for more xylitol dehydrogenase in the metabolic pathway, which in turn enhances ethanol production.

Substrate fermentation : The optimized conditions taken for fermentation of hydrolyzates of SCB and CC along with $1 \%$ urea were taken as fermentation medium. The ethanol produced by fermentation of SCB and CC is shown in Fig. 3A-3C. The concentration of ethanol produced by SCB and $\mathrm{CC}$ was 27.93 and $20.78 \mathrm{~g} \mathrm{l}^{-1}$. These results are significantly similar to the results of Patle and Lal (2007) and Latif and Rajoka (2001) who reported ethanol production by mixed cultures using agricultural wastes. The interference of intermediate compounds such as HMF, furfural in corn cob based agro-waste residue, when compared with sugarcane bagasse residues which automatically reduces the ethanol yield. However, according to Zhao et al. (2015), 

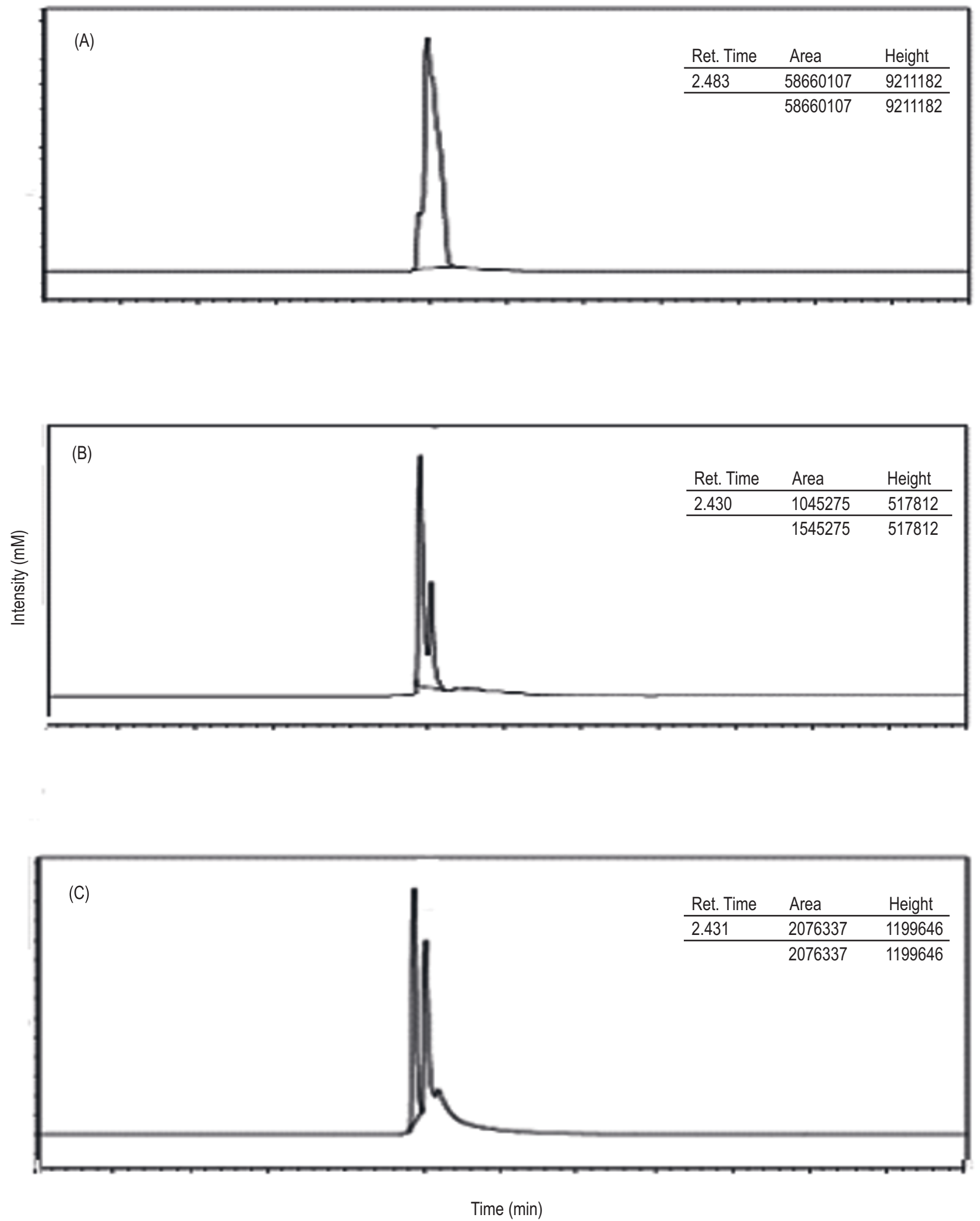

Fig. 3 : Gas Chromatogram showing the presence of ethanol in (A) reference standard; (B) corn cob and (C) sugarcane bagasse hydrolysate fermentation medium 
regardless of existing technologies for lignocellulosic biomass, bioethanol is not competent with fossil fuels when economic feasibility is considered.

The present study envisaged the possibilities of Bioethanol production from the agrowaste residues using indigenous yeast isolateCandida parapsilosis BKR1.The ethanol produced was found to be maximum $\left(27.93 \mathrm{~g} \mathrm{l}^{-1}\right)$ for SCB pretreated agro residue than for $\mathrm{CC}$ pretreated agro residue $\left(20.78 \mathrm{gl}^{-1}\right)$ at optimum conditions.

\section{References}

Bjerre, A.B., A.B Olesen, T. Fernqvist, A. Ploger and A.S. Schmidt: Pretreatment of wheat straw using combined wet oxidation and alkaline hydrolysis resulting in convertible cellulose and hemice1 lulose. Biotechnol Bioeng.,49, 568-577 (1996).

Cachet, T.: Guidelines for the quantitative gas chromatography of volatile flavoring substances, from the working group on methods of analysis of the international organization of the flavor industry (IOFI). Flavour Fragrance J.,26, 297-299 (2011).

Chan-u-tit, P., L. Laopaiboon, P. Jaisil and P. Laopaiboon: High level ethanol production by nitrogen and osmoprotectant supplementation under very high gravity fermentation conditions. Energies., 6, 884-899 (2013).

Du Preez, J.C., M. Bosch and B.A. Prior: The fermentation of hexose and pentose sugars by Candida shehatae and Pichia stipitis. App. Microbio. Biotechno.,23, 228-233 (1986).

Graves, T., N.V. Narendranath, K. Dawson and R. Power: Effect of pH and lactic or acetic acid on ethanol productivity by Saccharomyces cerevisiae in corn mash. $\mathrm{J}$ Ind. Microbio. Biotechnol.,33, 469-474 (2006).

Itelima, J., A. Ogbonna, S. Pandukur, J. Egbere and A. Salami: Simultaneous saccharification and fermentation of corn cobs to bio-ethanol by co-culture of Aspergillus niger and Saccharomyces cerevisiae. Int.J. Environ. Sci. Develop.,4, 239-242 (2013).

Izmirlioglu,G. and A. Demirci: Ethanol production from waste potato mash by using Saccharomyces cerevisiae. Appl. Sci., 2, 738-753 (2012).

Jeffries, T.W.: Effects of culture conditions on the fermentation of xylose to ethanol by Candida shehatae. Biotechno. Bioengin. Sympo.,15, 149-166 (1985).

Latif, F. and M.I. Rajoka: Production of ethanol and xylitol from corn cobs by yeasts. Bioresour. Technol.,77, 57-63 (2001).

Lin, Y. and S. Tanaka: Ethanol fermentation from biomass resources: Current state andprospects. Appl. Microbiol. Biotechnol., 69, 627-642(2006).

Martiniano, S.E., A.K. Chandel, L.C.S.R. Soares, F.C. Pagnocca and S.S. da Silva: Evaluation of novel xylose-fermenting yeast strains from Brazilian forests for hemicellulosic ethanol production from sugarcane bagasse. Biotechnology, 3, 345-352 (2013).

Miller, G.L.: Use of dinitrosalicylic acid reagent for determination of reducing sugar. Anal.Chem., 31, 426-428 (1959).

Mishra, J., D. Kumar, S. Samanta and M.K. Vishwakarma: A comparative study of ethanol production from various agro residues by using Saccharomyces cerevisiae and Candida albican. J. Yeast Fungal Res., 3, 12-17 (2012).

Mussatto, S.I., G. Dragone, P.M.R. Guimarães, J.P. Silva, L.M. Carneiro, I.C. Roberto, A. Vicente, L. Domingues and J.A. Teixeira: Technological trends, global market, and challenges of bio-ethanol production. Biotechnol.Adv.,28, 817-830 (2010).

Nofemele, Z., P. Shukla, A. Trussler, K. Permaul and S. Singh: Improvement of ethanol production from sugarcane molasses through enhanced nutrient supplementation using Saccharomyces cerevisiae. J. Brewing Distilling, 3, 29-35 (2012).

Osman, M.E., O.H. Khattab, I.A. Hammad and N.I. El-Hussieny: Optimization of bio-fuel production by Saccharomyces cerevisiae isolated from sugar cane bagasse. J. Amer. Sci., 7, 485-492 (2011).

Patle, S. and B. Lal: Ethanol production from hydrolysed agricultural wastes using mixed culture of Zymomonas mobilis and Candida tropicalis. Biotechnol. Lett.,29, 1839-1843 (2007).

Ray, R.C., S. Mohapatra, S. Panda and S. Kar: Solid substrate fermentation of cassava fibrous residue for production of aamylase, lactic acid and ethanol. J. Environ. Biol., 29, 111-115 (2008).

Turhan, I., K.L. Bialka, A. Demirci and M. Karhan: Ethanol production from carob extract by using Saccharomyces cerevisiae. Bioresour. Technol.,101, 5290-5296 (2010).

Verardi, A., A. Blasi, A.Molino, L. Albo and V. Calabrò: Improving the enzymatic hydrolysis of Saccharum officinarum $L$. bagasse by optimizing mixing in a stirred tank reactor: Quantitative analysis of biomass conversion. Fuel Process. Technol.,149, 15-22 (2016).

Zhao, L. X. Zhang, J. Xu, X. Ou, S. Chang, M. Wu: Techno-economic analysis of bioethanol production from lignocellulosic biomass in China: Dilute-acid pretreatment and enzymatic hydrolysis of corn stover. Energies, 8, 4096-4117 (2015). 